\title{
MRI-based biodistribution assessment of holmium-166 poly (L-lactic acid) microspheres after radioembolisation
}

\author{
Gerrit H. van de Maat • Peter R. Seevinck • \\ Mattijs Elschot • Maarten L. J. Smits • Hendrik de Leeuw • \\ Alfred D. van het Schip • Maarten A. D. Vente • \\ Bernard A. Zonnenberg • Hugo W. A. M. de Jong • \\ Marnix G. E. H. Lam • Max A. Viergever • \\ Maurice A. A. J. van den Bosch • Johannes F. W. Nijsen • \\ Chris J. G. Bakker
}

Received: 25 May 2012 /Revised: 20 July 2012 / Accepted: 15 August 2012 / Published online: 27 September 2012

(C) The Author(s) 2012. This article is published with open access at Springerlink.com

\begin{abstract}
Objectives To demonstrate the feasibility of MRI-based assessment of the intrahepatic Ho-PLLA-MS biodistribution after radioembolisation in order to estimate the absorbed radiation dose.

Methods Fifteen patients were treated with holmium-166 $\left({ }^{166} \mathrm{Ho}\right)$ poly(L-lactic acid)-loaded microspheres (Ho-PLLAMS, mean $484 \mathrm{mg}$; range $408-593 \mathrm{mg}$ ) in a phase I study. Multi-echo gradient-echo MR images were acquired from which $\mathrm{R}_{2}{ }^{*}$ maps were constructed. The amount of HoPLLA-MS in the liver was determined by using the relaxivity $\mathrm{r}_{2}{ }^{*}$ of the Ho-PLLA-MS and compared with the administered amount. Quantitative single photon emission computed tomography (SPECT) was used for comparison with MRI regarding the whole liver absorbed radiation dose.

Results $\mathrm{R}_{2}{ }^{*}$ maps visualised the deposition of Ho-PLLAMS with great detail. The mean total amount of Ho-PLLAMS detected in the liver based on MRI was $431 \mathrm{mg}$ (range $236-666 \mathrm{mg}$ ) or $89 \pm 19 \%$ of the delivered amount (correlation coefficient $r=0.7 ; P<0.01$ ). A good correlation was
\end{abstract}

G. H. van de Maat $(\bowtie) \cdot$ P. R. Seevinck $\cdot$ H. de Leeuw $•$

M. A. Viergever

Image Sciences Institute, University Medical Center Utrecht, Q S.459, PO Box 85500, 3508 GA Utrecht, The Netherlands e-mail: g.h.vandemaat@umcutrecht.nl

M. Elschot • M. L. J. Smits • A. D. van het Schip •

M. A. D. Vente $\cdot$ B. A. Zonnenberg $\cdot$ H. W. A. M. de Jong $\cdot$

M. G. E. H. Lam - M. A. A. J. van den Bosch - J. F. W. Nijsen •

C. J. G. Bakker

Department of Radiology and Nuclear Medicine,

University Medical Center Utrecht,

Utrecht, The Netherlands found between the whole liver mean absorbed radiation dose as assessed by MRI and SPECT (correlation coefficient $r=0.927 ; P<0.001)$.

Conclusion MRI-based dosimetry for holmium-166 radioembolisation is feasible. Biodistribution is visualised with great detail and quantitative measurements are possible.

Key Points

- Radioembolisation is increasingly used for treating unresectable primary or metastatic liver tumours.

- MRI-based intrahepatic microsphere biodistribution assessment is feasible after holmium-166 radioembolisation.

- MRI enables quantification of holmium-166 microspheres in liver in a short imaging time.

- MRI can estimate the whole liver absorbed radiation dose following holmium-166 radioembolisation.

Keywords Radioembolisation · Holmium · MRI ·

Dosimetry $\cdot$ Biodistribution

\section{Introduction}

Yttrium-90 $\left({ }^{90} \mathrm{Y}\right)$ radioembolisation (RE) is increasingly used for the treatment of patients with unresectable primary or metastatic liver tumours [1-6]. The two commercially available products, SIR-Spheres ${ }^{\circledR}$ (Sirtex Medical Limited, North Sydney, NSW, Australia) and TheraSphere ${ }^{\circledR}$ (Nordion, Ottawa, Canada), use empirical methods for dose calculation, based on body surface area and total liver weight respectively. However, apart from the total dose delivered to the liver, the efficacy of RE will largely depend on the intrahepatic dose distribution or, more specifically, on the ratio between the tumour absorbed dose and healthy tissue absorbed dose. 
Quantitative assessment of the post-administration intrahepatic microsphere distribution is therefore indispensable for evaluation of RE toxicity and efficacy and can potentially be used for the prediction of patient response and patient-specific therapeutic dose optimisation. Image-based approaches to assess microsphere distribution after RE have gained interest but are mostly hampered by the limited imaging possibilities of the isotope ${ }^{90} \mathrm{Y}$. Quantitative ${ }^{90} \mathrm{Y}$-SPECT imaging has limited spatial resolution because it is based on ${ }^{90} \mathrm{Y}$ Bremsstrahlung [7-10], whereas ${ }^{90}$ Y-PET has better spatial resolution but low sensitivity $[11,12]$. As a consequence, alternative methods of visualising the microspheres have been investigated, such as MR imaging of iron-labelled microspheres [13].

In the past decade, holmium-166 $\left({ }^{166} \mathrm{Ho}\right)$ poly(L-lactic acid)-loaded microspheres (Ho-PLLA-MS) have been developed as a potential radioembolisation particle $[14,15]$. Neutron activated holmium-166 is a $\beta$-emitting $\left(E_{\max }=1.77\right.$ and $1.854 \mathrm{MeV} ; \mathrm{I}_{\beta}=48.7 \%$ and $50 \%$, respectively; $\mathrm{T}_{1 / 2}=$ $26.83 \mathrm{~h})$ and $\gamma$-emitting $\left(\mathrm{E}_{\gamma}=80.6 \mathrm{keV}\right)$ lanthanide that, embedded in microspheres of poly(L-lactic acid), yields a particle suitable for internal radiation therapy that can be visualised with a range of clinical imaging techniques, including SPECT and MRI. This multimodal imaging has previously been demonstrated in preclinical animal studies [16-18]. It was also shown that MRI combines high sensitivity with high spatialtemporal resolution and with superior soft tissue contrast [19] and thus can be used to cover a broad range of clinically interesting imaging parameters. More recently, it was shown for an ex vivo situation that MRI is able to provide a good measurement of the Ho-PLLA-MS radiation-absorbed dose by convolution of quantitative MRI data with a ${ }^{166} \mathrm{Ho}$ dose point kernel [20].

The feasibility of image-guided RE using Ho-PLLA-MS was tested in a first-time-in-man clinical phase I study. The aim of the work described in this article was to investigate the capability of MRI to measure the intrahepatic microsphere distribution in order to quantify the absorbed radiation dose in patients treated with Ho-PLLA-MS radioembolisation.

\section{Materials and methods}

\section{Patients}

To be eligible to enter the phase I study each patient needed to comply with the following inclusion criteria: written informed consent; age 18 years or older; presence of liverdominant, unresectable, chemorefractory liver metastases of any primary tumour with at least one measurable lesion of $\geq 10 \mathrm{~mm}$ in the longest diameter on $\mathrm{CT}$; an estimated life expectancy $>3$ months; World Health Organisation (WHO) performance status $0-2$; and a negative pregnancy test for women. Furthermore, patients with contraindications for
MRI were excluded from treatment. Ethics approval for this study was obtained from the institutional review board; the study was performed in accordance with the Declaration of Helsinki and was registered with Clinicaltrials.gov, no. NCT01031784.

\section{Treatment}

Holmium-165 poly(L-lactic acid) microspheres with a mean diameter of $30 \mu \mathrm{m}$ (range $20-50 \mu \mathrm{m}$ ) and a holmium content of $18.7 \%$ (weight/weight) were prepared under Good Laboratory Practice (GLP) guidelines as previously described [14]. The amount of microspheres $(600 \mathrm{mg})$ was weighed, packed in high-density polyethylene vials (Posthumus Plastics, Beverwijk, The Netherlands) and sent to the nuclear reactor (Delft University of Technology, Delft, The Netherlands) for neutron activation [21]. The calculation of the amount of radioactivity was based on liver weight, conforming to the clinically used approach for TheraSphere ${ }^{\circledR}$. The amount of administered ${ }^{166} \mathrm{Ho}$-radioactivity was calculated using the target liver absorbed dose in a dose-escalation fashion $(20,40,60$, or $80 \mathrm{~Gy})$ as previously described [22]. All patients received whole liver treatment. The activity to be administered was calculated assuming homogeneous distribution of the activity in the liver using the following formula: $A_{\text {Hol66 }}(M B q)=$ Liver Dose $\times 63$ $(M B q / J) \times L W$ where $A_{H o l 66}$ is the administered activity, $L W$ is the liver weight in $\mathrm{kg}$, and Liver Dose is the intended liver absorbed radiation dose in Gy. While the radioactivity was different for each patient, the amount of administered Ho-PLLA-MS was kept constant at $600 \mathrm{mg}$. Before administration, the amount of radioactivity in the vials was measured using a dose calibrator (VDC-404, Veenstra Instrumenten B.V., Joure, The Netherlands), and the specific activity of the Ho-PLLA-MS was determined. After administration, activity measurements were performed on the administration system to determine the amount of microspheres that was actually delivered to the subjects retrospectively.

\section{Radioembolisation procedures}

Radioembolisation was performed according to standard recommendations [23, 24]. Relevant vessels in particular branches of the hepatic artery supplying organs other than the liver, e.g., the gastroduodenal artery (GDA) and right gastric artery (RGA), were coiled during a pre-treatment angiographic procedure to prevent extrahepatic deposition of activity. A scout dose of ${ }^{99 \mathrm{~m}}$ Tc-MAA $(150 \mathrm{MBq}, 0.8 \mathrm{mg}$, TechneScan LyoMaa ${ }^{\circledR}$, Mallinckrodt Medical B.V., Petten, The Netherlands) was injected into the hepatic artery followed by planar gamma camera imaging and SPECT to check for inadvertent extrahepatic deposition. The ${ }^{99 \mathrm{~m}} \mathrm{Tc}-\mathrm{MAA}$ lung shunt was measured by planar scintigraphic imaging, and 
shunt fractions were determined by region of interest (ROI) analyses [25]. If the lung shunt fraction of ${ }^{99 \mathrm{~m}} \mathrm{Tc}-\mathrm{MAA}$ was $<20 \%$ and no other extrahepatic deposition of ${ }^{99 \mathrm{~m}} \mathrm{Tc}-\mathrm{MAA}$ was detected, treatment with Ho-PLLA-MS was performed within 2 weeks.

\section{Data acquisition for dosimetry}

For intrahepatic biodistribution assessment of the microspheres, abdominal SPECT and MRI were performed.

\section{$M R I$}

Magnetic resonance images were acquired 1-2 weeks before and 1 week after administration of the therapeutic dose of Ho-PLLA-MS. Since MRI utilises the paramagnetic nature of the microspheres rather than the radioactivity, the post-therapy imaging time point was of minor relevance, and post-therapy images were acquired during the patients' first weekly visit at the outpatient clinic after treatment. Imaging was performed using a 1.5-T whole body system (Achieva, Philips Healthcare, Best, The Netherlands) equipped with a 16-element torso coil. For detection and quantification of Ho-PLLA-MS, a multi-slice multi-gradient echo (MGE) sequence was used, acquiring 16 echoes during breath hold with an in-plane resolution of $2 \times 2 \mathrm{~mm}^{2}$ and a slice thickness of $6 \mathrm{~mm}$. Imaging parameters included: FOV: $288 \times 384 \mathrm{~mm}^{2}$, number of slices: 45 , TR/TE1/ $\Delta$ TE: $440 \mathrm{~ms} /$ $1.33 \mathrm{~ms} / 1.15 \mathrm{~ms}$, flip angle: $50^{\circ}$. Sensitivity encoding (SENSE) with a factor of 2.5 was used for acceleration resulting in an imaging time of $3 \times 19 \mathrm{~s}$ during breath hold. For anatomical information and segmentation purposes, $\mathrm{T}_{2}$ weighted turbo spin echo (TSE) images were acquired with identical FOV and voxel size. Imaging parameters included: TR/TE: $830 \mathrm{~ms} / 80 \mathrm{~ms}$; SENSE factor: 2 ; imaging time: $2 \times$ $19 \mathrm{~s}$ during breath hold. In addition, $\mathrm{T}_{1}$-weighted turbo field echo (TFE) images were acquired using an in-plane resolution of $1.8 \times 3.6 \mathrm{~mm}^{2}$; slice thickness: $10 \mathrm{~mm}$; TR/TE: $8.5 \mathrm{~ms} /$ $4.18 \mathrm{~ms}$; total imaging time: $2 \times 14 \mathrm{~s}$ during breath hold.

To determine the sensitivity of MRI for Ho-PLLA-MS, as expressed by the $\mathrm{r}_{2}{ }^{*}$ relaxivity (relaxation rate per $\mathrm{mg} / \mathrm{ml}$ Ho-PLLA-MS), a calibration phantom setup containing known microsphere concentrations $(0,2.1,4.1,6.1$ and $8 \mathrm{mg} / \mathrm{ml} \mathrm{Ho-PLLA-MS}$ in agarose gel) was imaged using the MGE sequence described above.

\section{SPECT}

Single photon emission computed tomography images were acquired 3-6 days after administration of Ho-PLLA-MS. The imaging time point did depend on the total activity that was administered to the patient and was based on the maximum tolerated count rate of the SPECT system to acquire images without dead-time effects. The first nine patients underwent imaging using a FORTE ${ }^{\mathrm{TM}}$ dual-headed gamma camera (FORTE $^{\text {TM }}$, Philips Medical Systems, Milpitas, CA, USA) equipped with gadolinium-153 scanning line sources for transmission CT. The last six patients underwent imaging using a Siemens Symbia T16 SPECT/CT system (Siemens, Erlangen, Germany), which combines a dual-headed gamma camera with a 16-slice CT system. Medium energy collimators were used on both systems. Energy windows were set at $80.6 \mathrm{keV}$ (15\% window width) for the holmium166 photo peak and at $118 \mathrm{keV}$ (12\% window width) for the correction of downscatter. One hundred twenty projections of $30 \mathrm{~s}$ were acquired in a $180^{\circ}$ (FORTE) or $360^{\circ}$ (Symbia T16) orbit around the liver. Quantitative image data were reconstructed to a $128^{3}$ matrix size with an isotropic voxel size of $4.7 \mathrm{~mm}^{3}$ (FORTE) or $4.8 \mathrm{~mm}^{3}$ (Symbia T16), using an ordered subsets expectation maximisation algorithm including a hybrid scatter correction method [26], resulting in an absolute quantitative 3D activity distribution in $\mathrm{MBq} / \mathrm{voxel}$.

\section{Data analyses}

Magnetic resonance images were processed using software code written in MATLAB (MathWorks, Natick, MA, USA). From the MGE MRI patient data, $\mathrm{R}_{2}{ }^{*}$ values were estimated voxelwise using a mono-exponential fitting algorithm weighting all signal amplitudes equally. To minimise the influence of noise, signal intensities lower than $3 \sigma$ were excluded from the fitting procedure, with $\sigma$ the standard deviation (SD) of the signal determined in a region with homogeneous signal intensity and free from Ho-PLLA-MS. $\mathrm{R}_{2}{ }^{*}$ values were determined for data obtained both before and after RE. For further analysis, the liver was manually segmented based on the tissue contrast of the corresponding $\mathrm{T}_{2}$-weighted TSE images. The distribution of estimated liver $\mathrm{R}_{2}{ }^{*}$ values was measured, and the mean $\mathrm{R}_{2}{ }^{*}$ value together with the SD was calculated.

To determine the change in $\mathrm{R}_{2}{ }^{*}\left(\Delta \mathrm{R}_{2}{ }^{*}\right)$ after therapy, a baseline $\mathrm{R}_{2}{ }^{*}$ value determined by the mean $\mathrm{R}_{2}{ }^{*}$ of the liver before Ho-PLLA-MS administration was subtracted from the post-therapy $\mathrm{R}_{2}{ }^{*}$ maps. Voxelwise concentrations of Ho-PLLA-MS were determined from the $\Delta \mathrm{R}_{2}{ }^{*}$ maps by the relationship [Ho-PLLA-MS] $=\Delta \mathrm{R}_{2}{ }^{*} / \mathrm{r}_{2}{ }^{*}$, with $\mathrm{r}_{2}{ }^{*}$ measured from the calibration phantom setup. Using the voxel volume and the total volume included in the segmentation, the total amount of Ho-PLLA-MS in the liver was determined.

For all patients, the MRI-based amount was compared with the total amount that was assumed to be delivered to the liver. This amount was determined by correcting the delivered amount of Ho-PLLA-MS by the ${ }^{99 \mathrm{~m}} \mathrm{Tc}-\mathrm{MAA}$ lung shunt fraction and the amount of Ho-PLLA-MS left in the 
administration system. Data from patients who had surgical clips implanted (as a consequence of former partial liver resection) were separated from the patients without clips, and means were also compared separately for these two groups. This separation was made because metallic surgical clips are known to distort gradient echo images [27-29] and hence were suspected to influence MRI-based quantification.

As a final step, the mean radiation absorbed dose was calculated on a whole liver level using MRI and SPECT dose maps that were constructed using the method previously described for an ex-vivo liver model [20]. In short, MRI concentration values were converted into $\mathrm{MBq} / \mathrm{voxel}$ using the voxel size and the specific activity of the microspheres $(\mathrm{MBq} / \mathrm{mg})$ at the moment of injection. The SPECT reconstructions provided an absolute quantitative activity in $\mathrm{MBq} /$ voxel. Both MRI and SPECT activity maps were convolved with a ${ }^{166} \mathrm{Ho}$ dose point kernel that was calculated using the Monte Carlo code MCNPX (vs. 2.5.0; LANL, Los Alamos, NM, USA) according to the method described in Medical Internal Radiation Dose (MIRD) Pamphlet 17 [30], assuming a tissue density for liver tissue of $1.06 \mathrm{~g} / \mathrm{cm}^{3}$ according to International Commission on Radiation Units and Measurements (ICRU) Report 44 [31]. The voxel size of the dose point kernel was equal to the native resolution of the image technique $(2 \times 2 \times 6 \mathrm{~mm}$ for MRI, $4.7 \mathrm{~mm}$ isotropic for SPECT). After manual co-registration of SPECT maps to MRI and segmentation, mean liver absorbed dose values were calculated for both techniques.

\section{Statistical analysis}

Descriptive statistics of means, standard deviations and ranges were calculated for continuous variables. The paired Student's $t$-test was used for comparison of means (two-tailed $95 \%$ confidence interval). Bivariate Pearson's correlation (one-tailed $95 \%$ confidence interval) was used for correlation between Ho-PLLA-MS calculations based on MRI and the actual amount for the whole liver and the correlation between MRI and SPECT dosimetry on a whole liver level. SPSS software (SPSS for Windows, version 13.0; SPSS Inc., Chicago, IL, USA) was used for all analyses.

\section{Results}

Fifteen patients were included in this phase I dose-escalation study. Nine men and six women, median age 55 years (range 38-87 years), were treated with escalating whole liver doses of 20-80 Gy. The median number of liver tumours was 5 (range 1-21), with a median fractional liver involvement of $14 \%$ (range $2-52 \%$ ), originating from an ocular melanoma ( 7 patients), colorectal carcinoma (5 patients), cholangiocarcinoma (2 patients) or breast carcinoma (1 patient). Five patients had surgical clips implanted in the liver as a consequence of former partial liver resection (number of clips ranging from 1 to $>20$ ). MRI data were analysed for 14 patients. One patient was excluded from MRI analysis because of incomplete data. The patients received a mean of $523 \mathrm{mg}$ Ho-PLLA-MS (range 438-640 mg). Corrected for shunting to the lungs (mean $7.4 \%$; range 3.0-13.0 \%), the mean administered amount of Ho-PLLA-MS to the liver was $484 \mathrm{mg}$ (range 408-593 mg; Table 1).

Ho-PLLA-MS caused enhanced MR signal decay after administration, translating into high $\mathrm{R}_{2}{ }^{*}$ values (Fig. 1). Strongly increased $\mathrm{R}_{2}{ }^{*}$ values were observed locally, corresponding to tumour lesions with high signal intensity on $\mathrm{T}_{2}$-weighted images. Histograms of the $\mathrm{R}_{2}{ }^{*}$ values found in the liver showed an overall shift of $R_{2}{ }^{*}$ values toward higher values and a broadening of the $\mathrm{R}_{2}{ }^{*}$ distribution after treatment (Fig. 2).

The mean $\mathrm{R}_{2}{ }^{*}$ values, measured over the entire liver for all patients before treatment (mean $31.5 \mathrm{~s} \mathrm{~s}^{-1}$; range 27.9$37.3 \mathrm{~s}^{-1}$ ), significantly increased after administration of HoPLLA-MS (mean $52.7 \mathrm{~s}^{-1}$; range 41.6-78.2 $\mathrm{s}^{-1} ; P<0.001$; Table 1).

From the phantom setup, an $r_{2}{ }^{*}$ relaxivity of $103 \pm$ $4 \mathrm{~s}^{-1} \cdot \mathrm{mg}^{-1} \cdot \mathrm{ml}$ was measured for the used Ho-PLLA-MS at $1.5 \mathrm{~T}$, which is in good agreement with the values in the literature $[19,20]$. This value was subsequently used to calculate Ho-PLLA-MS concentration maps. The concentration maps were comparable to the $\mathrm{R}_{2}{ }^{*}$ maps with relatively high concentrations found at tumour sites (Fig. 3). From the concentration maps and the volume of the liver, the amount of Ho-PLLA-MS was calculated. The mean total amount of Ho-PLLA-MS that was detected in the liver based on MRI was $431 \mathrm{mg}$ (range 236-666 mg). For all patients this was $89 \pm 19 \%($ mean \pm SD) of the amount delivered to the liver (correlation coefficient $r=0.7 ; P<0.01$ ). By excluding the data of the patients with surgical clips implanted in the liver, the mean MRI-based detected fraction increased to $96 \pm 13 \%$ (mean $\pm \mathrm{SD}$; correlation coefficient $r=0.8 ; P<0.01$; Table 1). MRI showed that the presence of surgical clips in the liver led to undetermined areas (black spots) surrounded by (virtual) high concentration values (Fig. 4). This led to a poor correlation between Ho-PLLA-MS amounts calculated on MRI and the actual delivered amount of Ho-PLLA-MS (correlation coefficient $r=0.3 ; P=0.29$ ).

A good correlation was found between the whole liver mean absorbed radiation dose as assessed by MRI and SPECT (correlation coefficient $r=0.93 ; P<0.001$ including all patients, correlation coefficient $r=0.98 ; P<0.001$ excluding patients with surgical clips, correlation coefficient $r=0.81 ; P=0.10$ for only patients with clips). 
Table 1 Acquired biodistribution data of all 15 patients included in the phase I dose-escalation study (20, 4060 and 80 Gy). One patient was excluded from MRI analyses because of incomplete data. Data of patients with surgical clips present in the liver are shaded grey.
Administered amounts (admin Ho-PLLA-MS) were determined based on activity measurements of the administration system and lung shunt fraction. $\mathrm{R}_{2}{ }^{*}$, concentration and absorbed dose values are mean values over the entire liver

\begin{tabular}{|c|c|c|c|c|c|c|c|c|c|c|}
\hline \multicolumn{11}{|c|}{ Detection of Ho-PLLA-MS in the whole liver } \\
\hline \multirow{2}{*}{$\begin{array}{l}\text { Subject } \\
1\end{array}$} & \multirow{2}{*}{$\begin{array}{l}\begin{array}{l}\text { Dose cohort } \\
\text { (Gy) }\end{array} \\
20\end{array}$} & \multirow{2}{*}{$\begin{array}{l}\begin{array}{l}\text { No. of } \\
\text { surgical } \\
\text { clips }\end{array} \\
-\end{array}$} & \multirow{2}{*}{$\begin{array}{l}\text { Admin. Ho- } \\
\text { PLLA-MS } \\
(\mathrm{mg}) \\
490\end{array}$} & \multirow{2}{*}{$\begin{array}{l}\mathrm{R}_{2} * \text { pre } \pm \\
\mathrm{SD}\left(\mathrm{s}^{-1}\right) \\
\text { excl }\end{array}$} & \multirow{2}{*}{$\begin{array}{l}\mathrm{R}_{2}^{*} \text { post } \pm \\
\mathrm{SD}\left(\mathrm{s}^{-1}\right) \\
\\
\text { excl }\end{array}$} & \multirow{2}{*}{$\begin{array}{l}\text { Concentration } \\
\text { Ho-PLLA- } \\
\mathrm{MS} \pm \mathrm{SD} \\
(\mathrm{mg} / \mathrm{ml}) \\
\text { excl }\end{array}$} & \multicolumn{2}{|c|}{$\begin{array}{l}\text { MRI-based } \\
\text { Ho-PLLA-MS } \\
\text { in mg } \\
(\% \text { of admin })\end{array}$} & \multirow{2}{*}{$\begin{array}{l}\text { MRI- } \\
\text { based } \\
\text { absorbed } \\
\text { dose (Gy) } \\
\text { excl }\end{array}$} & \multirow{2}{*}{$\begin{array}{l}\begin{array}{l}\text { SPECT- } \\
\text { based } \\
\text { absorbed } \\
\text { dose (Gy) }\end{array} \\
9.5\end{array}$} \\
\hline & & & & & & & excl & excl & & \\
\hline 2 & 20 & - & 408 & $28.4 \pm 12.4$ & $43.3 \pm 21.8$ & $0.14 \pm 0.21$ & 467 & (115) & 12.4 & 9.1 \\
\hline 3 & 20 & $5-10$ & 449 & $31.1 \pm 18.6$ & $42.4 \pm 36.2$ & $0.11 \pm 0.35$ & 287 & $(64)$ & 6.6 & 12.5 \\
\hline 4 & 20 & - & 465 & $31.8 \pm 16.4$ & $45.4 \pm 21.2$ & $0.13 \pm 0.21$ & 452 & (97) & 16.4 & 14.1 \\
\hline 5 & 20 & - & 489 & $37.3 \pm 24.1$ & $61.4 \pm 39.7$ & $0.23 \pm 0.39$ & 415 & (85) & 13.4 & 11.0 \\
\hline 6 & 20 & - & 461 & $30.6 \pm 14.8$ & $59.2 \pm 23.6$ & $0.28 \pm 0.23$ & 447 & (97) & 15.9 & 11.9 \\
\hline 7 & 40 & $10-15$ & 411 & $33.2 \pm 20.3$ & $52.2 \pm 36.7$ & $0.18 \pm 0.36$ & 291 & (71) & 19.8 & 22.2 \\
\hline 8 & 40 & - & 472 & $33.9 \pm 19.7$ & $52.7 \pm 36.2$ & $0.19 \pm 0.35$ & 351 & (74) & 19.4 & 20.9 \\
\hline 9 & 40 & $10-15$ & 453 & $30.9 \pm 21.7$ & $50.2 \pm 29.8$ & $0.28 \pm 0.23$ & 495 & (109) & 29.5 & 27.7 \\
\hline 10 & 60 & - & 501 & $27.9 \pm 15.7$ & $42.2 \pm 21.4$ & $0.14 \pm 0.21$ & 436 & (87) & 32.4 & 36.6 \\
\hline 11 & 60 & - & 485 & $31.4 \pm 19.6$ & $53.8 \pm 32.2$ & $0.23 \pm 0.30$ & 457 & (94) & 41.3 & 36.9 \\
\hline 12 & 60 & $>20$ & 478 & $35.8 \pm 34.2$ & $45.3 \pm 30.0$ & $0.09 \pm 0.29$ & 236 & (49) & 19.6 & 37.3 \\
\hline 13 & 80 & - & 593 & $29.2 \pm 13.8$ & $69.4 \pm 56.4$ & $0.39 \pm 0.55$ & 597 & (101) & 59.3 & 53.1 \\
\hline 14 & 80 & 1 & 522 & $25.5 \pm 21.0$ & $41.6 \pm 25.7$ & $0.16 \pm 0.25$ & 442 & (85) & 42.0 & 41.3 \\
\hline 15 & 80 & - & 591 & $33.7 \pm 16.8$ & $78.2 \pm 54.8$ & $0.43 \pm 0.53$ & 666 & (113) & 68.8 & 55.2 \\
\hline
\end{tabular}
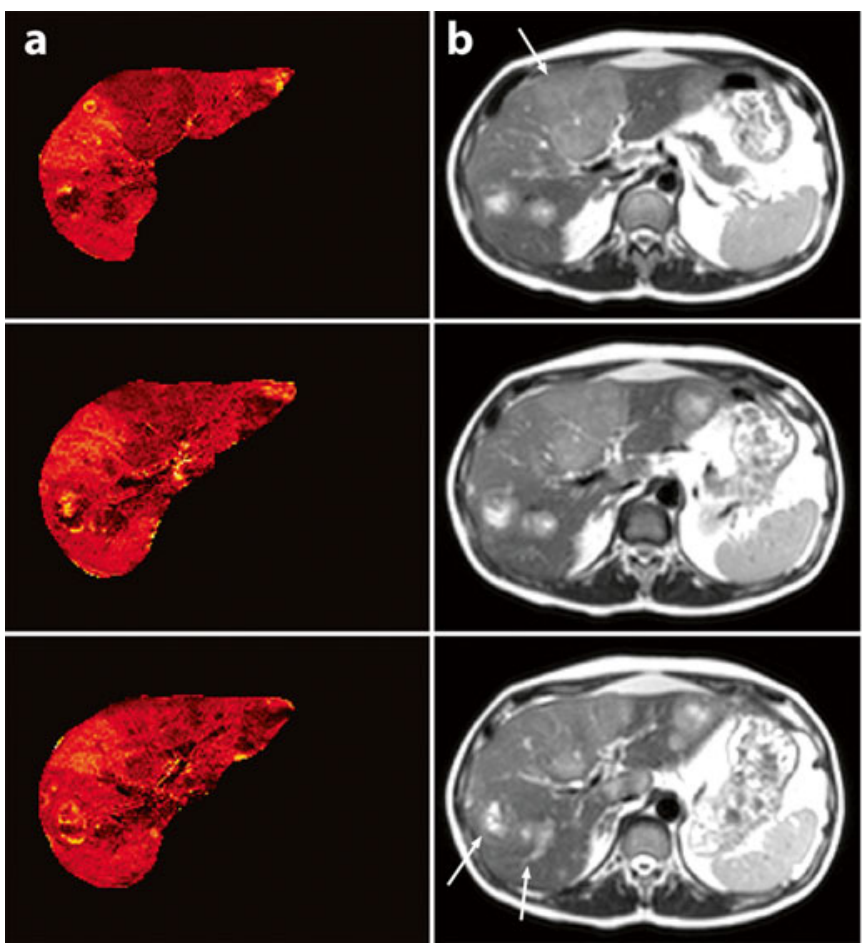

Fig. $1 \mathrm{R}_{2}{ }^{*}$ maps and SE images (TR/TE: $830 \mathrm{~ms} / 80 \mathrm{~ms}$, flip angle: $\left.90^{\circ}\right)$ before $(\mathrm{a}+\mathrm{b})$ and after $(\mathrm{c}+\mathrm{d}) \mathrm{RE}$. Variations in $\mathrm{R}_{2}{ }^{*}$ values before $\mathrm{RE}$ (a) correspond to variations on SE images (b). After RE, increased
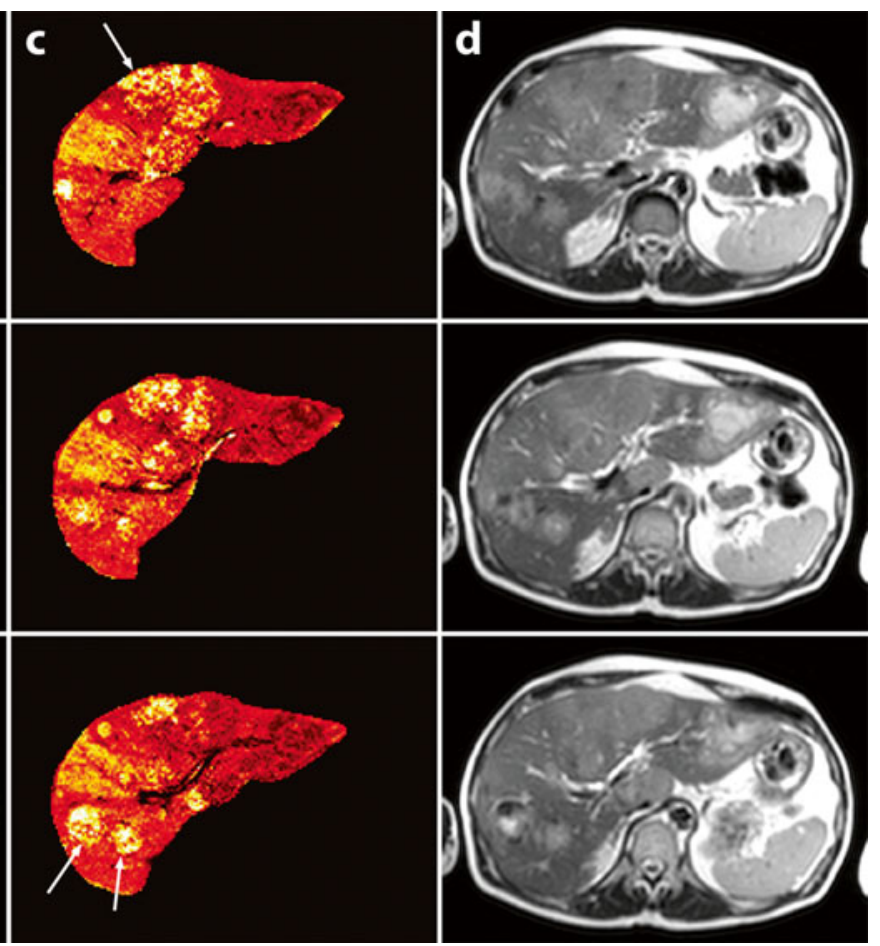

$\mathrm{R}_{2}{ }^{*}$ values were observed at locations with Ho-PLLA-MS deposition (c), corresponding to tumour lesions with high intensities on SE images (arrows) (d) 


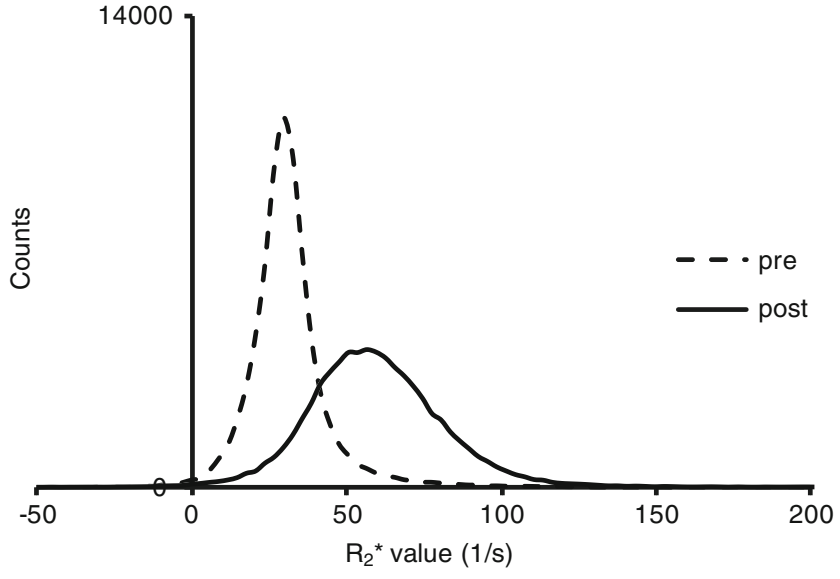

Fig. 2 Typical histogram of $\mathrm{R}_{2}{ }^{*}$ values measured in the whole liver of a patient before $(--)$ and after (-) adminstration of Ho-PLLA-MS. After administration the curves shifted toward higher values and a broader distribution of $\mathrm{R}_{2}{ }^{*}$ values was observed

Magnetic resonance imaging displayed the local microsphere distribution in much more detail because of the relatively high in-plane resolution, for example revealing an absence of Ho-PLLA-MS in the central part of a furthermore highly targeted tumour in some cases. This situation was rather obscured in the corresponding SPECT images owing to the lower resolution and blurring effects (Fig. 5).

\section{Discussion}

In this article we presented the first in vivo quantitative MRI data of ${ }^{166} \mathrm{Ho}$-poly(L-lactic acid) microspheres for imageguided radioembolisation using MRI.

Three-dimensional MRI-based assessment of the intrahepatic biodistribution of Ho-PLLA-MS was feasible. HoPLLA-MS concentration maps, which were constructed by using a time series of $\mathrm{T}_{2}{ }^{*}$-weighted images, clearly showed the ability of MRI to visualise the intrahepatic biodistribution of the microspheres. Furthermore, MRI provided both a quantitative measure of the Ho-PLLA-MS distribution and anatomical information, enabling direct localisation of the microsphere deposition within the tissue. MRI detected $89 \%$ of the actual delivered Ho-PLLA-MS in the whole liver. The mean whole liver absorbed radiation dose calculated on MRI correlated very well with the absorbed radiation dose on SPECT. A more detailed intra-tumour biodistribution assessment was possible using MRI compared with SPECT because of the better spatial resolution of MRI. The quantitative nature of MRI-based biodistribution assessment of Ho-PLLA-MS allows for personalised dosimetry in vivo and may ultimately lead to optimisation of radioembolisation with regard to safety and efficacy.
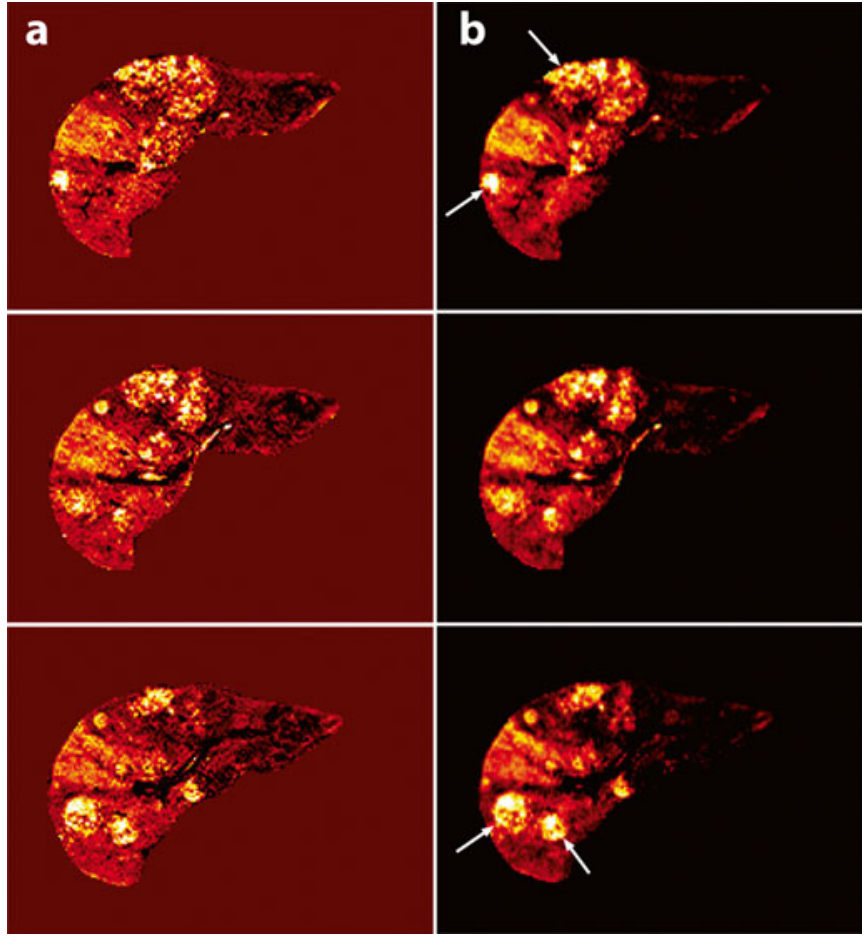

Fig. 3 MRI-based concentration maps (a) after Ho-PLLA-MS administration, MRI-based absorbed dose maps (b) together with their corresponding SPECT images (c) and $\mathrm{T}_{1}$-weighted images (d) (TR/ TE: $8.5 \mathrm{~ms} / 4.18 \mathrm{~ms}$, flip angle: $10^{\circ}$ ). The concentration maps were constructed from normalised $\mathrm{R}_{2}{ }^{*}$ maps by using the $\mathrm{r}_{2}{ }^{*}$ relaxivity of

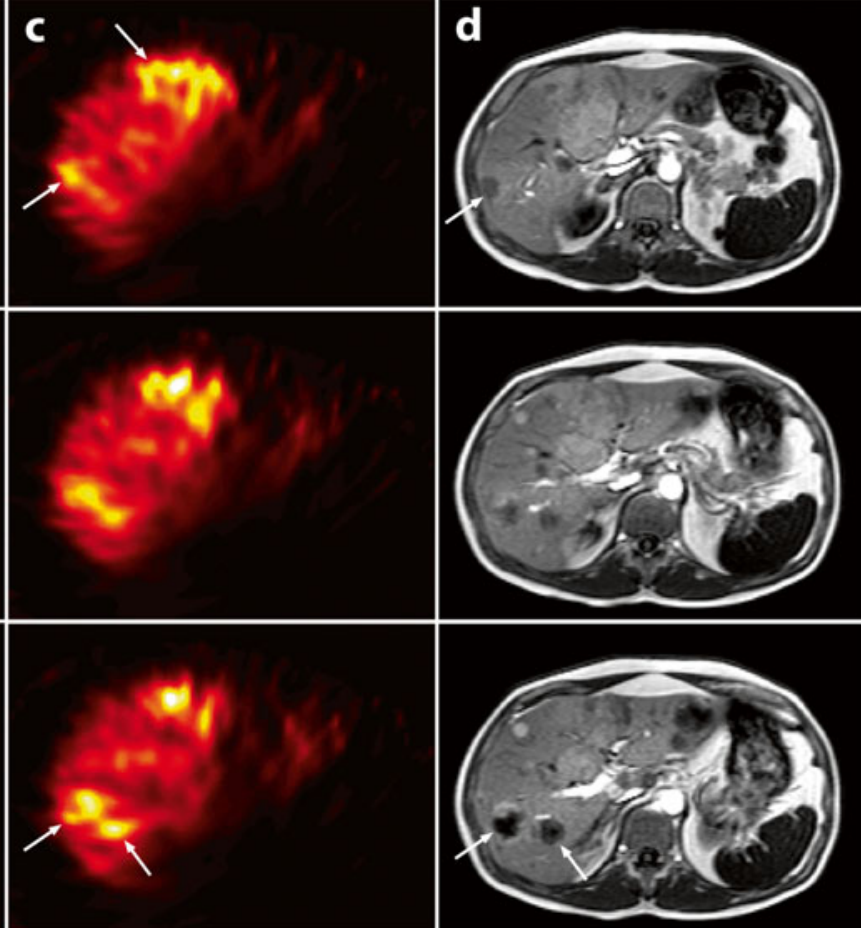

Ho-PLLA-MS. MRI-based dose maps were constructed by applying a dose point kernel after conversion of concentrations Ho-PLLA-MS to $\mathrm{MBq} /$ voxel. MRI-based concentration and absorbed dose correspond to the activity distribution on the SPECT images (c) and the tumour sites on $\mathrm{T}_{1}$-weighted images (d) (arrows) 
Fig. 4 MRI-based Ho-PLLAMS concentration maps before (a) and after (b) radioembolisation in a patient with multiple surgical clips after partial liver resection. At the location of the clips (arrows), quantification failed (black spots), while in the near vicinity extremely high concentration values were found (white rings)
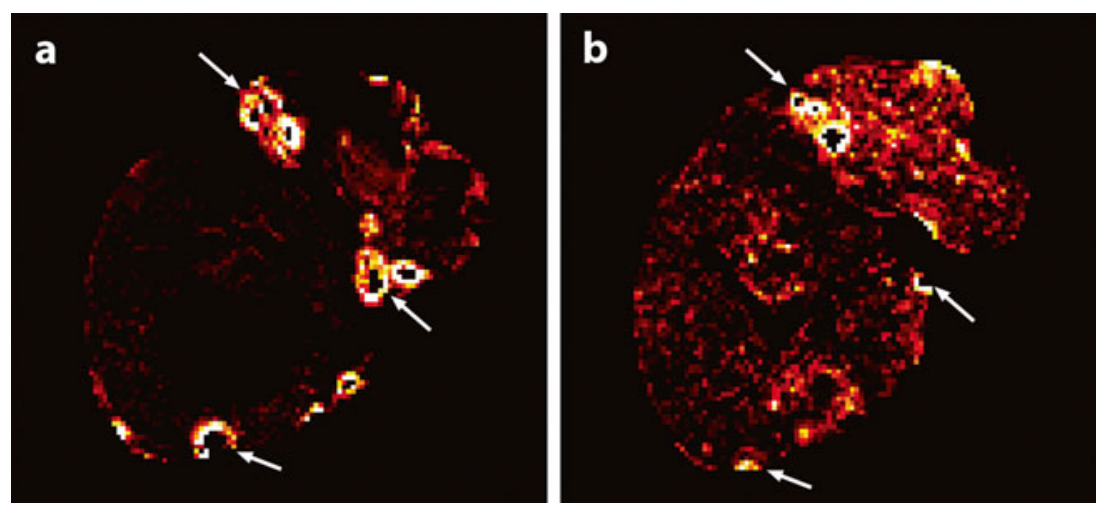

Compared with the actual administered amount of HoPLLA-MS in the liver, determined based on the injected amount and taking into account the ${ }^{99 \mathrm{~m}}$ Tc-MAA-based lung shunt fraction, the MRI quantification method presented in this work showed on average an underestimation. As shown, this was in part due to the presence of surgical clips (Fig. 4). These clips induced field distortions, resulting in large MR signal voids surrounded by large $\mathrm{R}_{2}{ }^{*}$ values. Therefore, locally the amount of Ho-PLLA-MS cannot be determined resulting in an underestimation of the microsphere amount in the vicinity of the clips. Although the presence of surgical clips does not necessarily lead to a contraindication for MRI-based dosimetry, biodistribution measurements in the vicinity of the clips should be interpreted with care. Another source of the discrepancy between the MRI-based amount and the amount delivered may be the use of ${ }^{99 \mathrm{~m}} \mathrm{Tc}$ MAA as a predictor of the lung shunt fraction. Although used to correct for extrahepatic deposition in order to estimate the Ho-PLLA-MS liver amount, it is known that, because of a number of dissimilarities between ${ }^{99 \mathrm{~m}} \mathrm{Tc}$ MAA
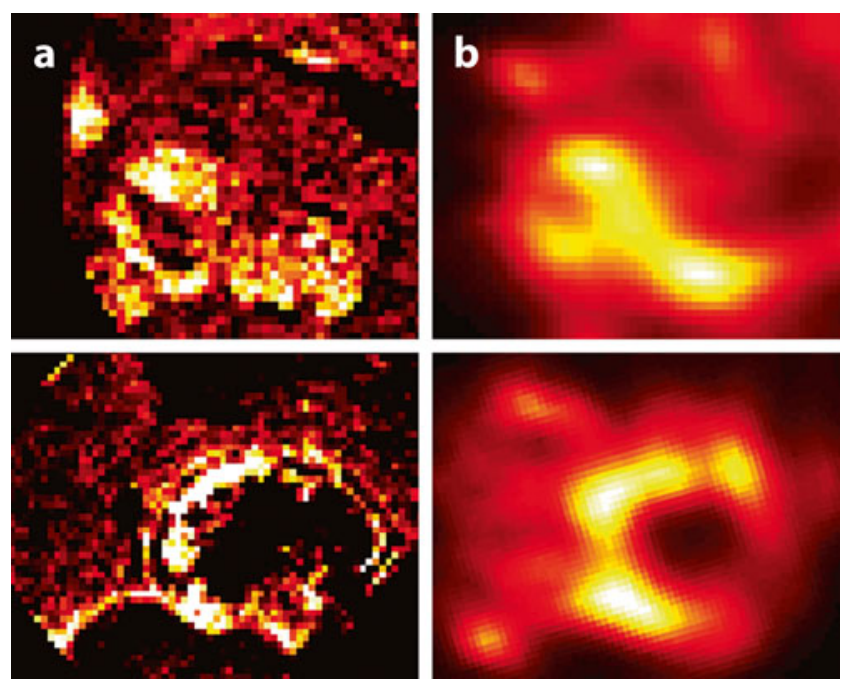

Fig. 5 Ho-PLLA-MS deposition around tumours. MRI-based concentration maps (a) show more detail (higher resolution) about the distribution of the microspheres than the SPECT-based activity maps. The Ho-PLLA-MS are primarily distributed in the peripheral parts of the tumour (a). This is less pronounced on the SPECT images (b) and Ho-PLLA-MS such as size, shape and density, ${ }^{99 \mathrm{~m}} \mathrm{Tc}$ MAA is not the optimal microsphere analogy [6]. Incorrect assessment of the lung shunt fraction may have led to an erroneous estimation of the amount of Ho-PLLA-MS delivered in the liver. This explanation is supported by the good correlation that was found between the MRI-based mean liver absorbed dose and the absorbed dose based on the more conventional method of image-based dosimetry, SPECT.

Quantification errors may also arise from the need to correct for baseline $\mathrm{R}_{2}{ }^{*}$ values in order to determine $\Delta \mathrm{R}_{2}{ }^{*}$. $\Delta \mathrm{R}_{2}{ }^{*}$ values were calculated by subtracting the mean liver $\mathrm{R}_{2}{ }^{*}$ value before administration from the post-treatment $\mathrm{R}_{2}{ }^{*}$ maps. Because of the variance of $\mathrm{R}_{2}{ }^{*}$ in the absence of microspheres as a result of tissue characteristics (Fig. 1), over- or underestimations will be introduced at the voxel level. The bias introduced in $\Delta \mathrm{R}_{2}{ }^{*}$ will depend on the local voxel $\mathrm{R}_{2}{ }^{*}$ value with respect to the mean $\mathrm{R}_{2}{ }^{*}$ over the whole VOI. In addition to these variations over space, changes may occur in tissue $T_{2}$ in the time interval between preand post-therapy imaging. Such changes may occur, for example, because of short-term therapy-induced oedema [32], which leads to increasing $T_{2}$ values, and thus decreasing $R_{2}$ values, owing to the higher water content as is generally reflected by hyperintense areas on $T_{2}$-weighted images [33]. A decrease in tissue $\mathrm{R}_{2}$ may therefore counteract the increase in $\mathrm{R}_{2}{ }^{*}$ because of Ho-PLLA-MS leading to an underestimation of Ho-PLLA-MS concentration.

To minimise these quantification errors, voxel-based subtraction of post- and pre-therapy maps seems to be crucial. Manual or (semi-) automatic registration of these maps may partly solve the problem, but a more robust solution will be to integrate MR imaging as part of the RE procedure. Data needed to construct the concentration maps shown in this work were acquired within $1 \mathrm{~min}$, and data processing can be performed within several minutes. Considering this short time scale, it is feasible to perform personalised MRI-based dosimetry during the actual RE procedure. This would enable fast verification and give the opportunity, if necessary, to immediately adjust the RE procedure. Moreover, MRI would enable monitoring of the administration of the Ho-PLLA-MS in real time. This has previously been demonstrated in a pig model [34]. 
The first MRI data of patients treated with ${ }^{166} \mathrm{Ho}$-poly (L-lactic acid) microspheres show the feasibility of MRIbased dosimetry for radioembolisation. Intrahepatic microsphere biodistribution can be visualised with great detail, and a good quantitative measurement of the absorbed radiation dose can be obtained within several minutes of the radioembolisation procedure. Biodistribution assessment in the vicinity of surgical clips should be interpreted with care.

Acknowledgments The patients described in this paper were treated in a phase I safety and toxicity study, the results of which are described in a separate paper.

This research project was funded by the Dutch Cancer Society (KWF Kankerbestrijding) under grant UU2009-4346 and by the Dutch Technology Foundation (STW) under grants UGT6069 and OTP06648. MLS is supported by a University Medical Center Utrecht Alexandre Suerman MD/PhD grant.

The authors thank Tjitske Bosma for her contribution to the study coordination and Remmert de Roos for his assistance in the preparation of the microspheres. Irradiation of the microspheres was performed by the Reactor Institute Delft, Delft, The Netherlands, under the auspices of M. Sarilar, M. Blaauw, H.T. Wolterbeek, R.J. Linssen and D.J. de Vries.

Open Access This article is distributed under the terms of the Creative Commons Attribution Noncommercial License which permits any noncommercial use, distribution, and reproduction in any medium, provided the original author(s) and the source are credited.

\section{References}

1. Salem R, Thurston KG (2006) Radioembolization with yttrium-90 microspheres: a state-of-the-art brachytherapy treatment for primary and secondary liver malignancies. J Vasc Interv Radiol 17: 1571-1594

2. Kennedy AS, Coldwell D, Nutting C et al (2006) Resin 90Ymicrosphere brachytherapy for unresectable colorectal liver metastases: modern USA experience. Int J Radiat Oncol Biol Phys 65:412-425

3. Vente MAD, Hobbelink MGG, van het Schip AD, Zonnenberg BA, Nijsen JFW (2007) Radionuclide liver cancer therapies: from concept to current clinical status. Anticancer Agents Med Chem 7:441-459

4. Gulec SA, Fong Y (2007) Yttrium 90 microsphere selective internal radiation treatment of hepatic colorectal metastases. Arch Surg 142:675-682

5. Vente MAD, Wondergem M, van der Tweel I et al (2009) Yttrium90 microsphere radioembolization for the treatment of liver malignancies: a structured meta-analysis. Eur Radiol 19:951-959

6. Bult W, Vente MAD, Zonnenberg BA, Nijsen JFW (2009) Microsphere radioembolzation of liver malignancies: current developments. Q J Nucl Med Mol Imaging 53:325-335

7. Fabbri C, Sarti G, Cremonesi M et al (2009) Quantitative analysis of $90 \mathrm{Y}$ Bremsstrahlung SPECT-CT images for application to 3D patient-specific dosimetry. Cancer Biother Radiopharm 24:145-154
8. Minarik D, Sjögreen-Gleisner K, Ljungberg M (2008) Evaluation of quantitative 90Y SPECT based on experimental phantom studies. Phys Med Biol 53:5689-5703

9. Minarik D, Sjögreen-Gleisner K, Linden O et al (2010) $90 \mathrm{Y}$ Bremsstrahlung imaging for abosrbed-dose assessment in highdose radioimmunotherapy. J Nucl Med 51:1974-1978

10. Elschot M, Nijsen JFW, Dam AJ, de Jong HWAM (2011) Quantitative evaluation of scintillation camera imaging characteristics of isotopes used in liver radioembolization. PLoS One 6:e26174

11. Lhommel R, van Elmbt L, Goffette P et al (2010) Feasibility of $90 Y$ TOF PET-based dosimetry in liver metastasis therapy using SIR-spheres. Eur J Nucl Med Mol Imaging 37:1654-1662

12. Gates VL, Esmail AAH, Marshall K, Spies S, Salem R (2011) Internal pair production of $90 \mathrm{Y}$ permits hepatic localization of microspheres using routine PET: proof of concept. J Nucl Med 52:72-76

13. Gupta T, Virmani S, Neidt TM et al (2008) MR Tracking of ironlabeled glass radioembolization microspheres during transcatheter delivery to rabbit VX2 liver tumors: feasibility study. Radiol 249:845-854

14. Nijsen JFW, Zonnenberg BA, Woittiez JRW et al (1999) Holmium-166 poly lactic acid microspheres applicable for intra-arterial radionuclide therapy of hepatic malignancies: effects of preparation and neutron activation techniques. Eur J Nucl Med 26:699-704

15. Nijsen JFW, van Steenbergen MJ, Kooijman H et al (2001) Characterization of poly(L-lactic acid) microspheres loaded with holmium acetylacetonate. Biomaterials 22:3073-3081

16. Nijsen JFW, Seppenwoolde JH, Havenith T, Bos C, Bakker CJG, van het Schip AD (2004) Liver tumors: MR imaging of radioactive holmium microspheres-phantom and rabbit study. Radiol 231:491-499

17. Seppenwoolde JH, Nijsen JFW, Bartels LW, Zielhuis SW, van het Schip AD, Bakker CJG (2005) Internal radiation therapy of liver tumors: qualitative and quantitative magnetic resonance imaging of the biodistribution of holmium-loaded microspheres in animal models. Magn Reson Med 53:76-84

18. Vente MAD, Nijsen JFW, de Wit TC et al (2008) Clinical effects of transcatheter hepatic arterial embolization with holmium-166 poly (L-lactic acid) microspheres in healthy pigs. Eur J Nucl Med Mol Imaging 7:1259-1271

19. Seevinck PR, Seppenwoolde JH, de Wit TC et al (2007) Factors affecting the sensitivity and detection limits of MRI, CT, and SPECT for multimodal diagnostic and therapeutic agents. Anticancer Agents Med Chem 7:317-334

20. Seevinck PR, van de Maat GH, de Wit TC, Vente MAD, Nijsen JFW, Bakker CJG (2012) Magnetic resonance imaging-based radiation-absorbed dose estimation of 166 -Ho microspheres in liver radioembolization. Int J Rad Oncol Biol Phys 83:e437-e444

21. Vente MA, Nijsen JF, de Roos R et al (2009) Neutron activation of holmium poly(L-lactic acid) microspheres for hepatic arterial radioembolization: a validation study. Biomed Microdevices 11:763-772

22. Smits MLJ, Nijsen JFW, van den Bosch et al (2010) Holmium166 radioembolization for the treatment of patients with liver metastases: design of the phase I HEPAR trial. J Exp Clin Cancer Res 29:70

23. Coldwell D, Sangro B, Wasan H, Salem R, Kennedy A (2011) General selection criteria of patients for radioembolization of liver tumors: an international working group report. Am J Clin Oncol 34:337-341

24. Kennedy A, Nag S, Salem R et al (2007) Recommendations for radioembolization of hepatic malignancies using ytrrium-90 microsphere brachytherapy: a consensus panel report from the radioembolization brachytherapy oncology consortium. Int J Radiat Oncol Biol Phys 68:13-23 
25. Leung WT, Lau WY, Ho SKW et al (1994) Measuring lung shunting in hepatocellular carcinoma with intrahepatic-arterial technetium-99 m macroaggregated albumin. J Nucl Med 35:70-73

26. de Wit T, Xiao J, Nijsen JF et al (2006) Hybrid scatter correction applied to quantitative holmium-166 SPECT. Phys Med Biol 51:4773-4787

27. Brown MA, Carden JA, Coleman RE, McKinney R Jr, Spicer LD (1987) Magnetic field effects on surgical ligation clips. Magn Reson Imag 5:443-453

28. Czervionke LF, Daniels DL, Wehrli FW et al (1988) Magnetic susceptibility artifacts in gradient-recalled echo MR imaging. Am J Neuroradiol 9:1149-1155

29. Port JD, Pomper MG (2000) Quantification and minimization of magnetic susceptibility artifacts on GRE images. J Comput Assist Tomogr 24:958-964
30. Bolch WE, Bouchet LG, Robertson JS et al (1999) MIRD pamphlet no. 17: the dosimetry of nonuniform activity distributionsradionuclide $\mathrm{S}$ values at the voxel level. Medical Internal Radiation Dose Committee. J Nucl Med 40:11S-36S

31. International Commission on Radiation Units and Measurements (1989) Tissue Substitutes in Radiation Dosimetry and Measurement. 44th edn. Bethesda, MD

32. Kamel IR, Bluemke DA (2002) Imaging evaluation of hepatocellular carcinoma. J Vasc Interv Radiol 13:S173-S183

33. Rummeny E, Weissleder R, Stark DD et al (1989) Primary liver tumours: diagnosis by MR imaging. Am J Roentgenol 152:63-72

34. Seppenwoolde JH, Bartels LW, van der Weide R, Nijsen JFW, van het Schip AD, Bakker CJG (2006) Fully MR-guided hepatic artery catheterization for selective drug delivery: a feasibility study in pigs. J Magn Reson Imaging 23:123-129 